\title{
Metabolic control analysis
}

\section{An application of signal flow graphs}

\author{
Asok K. SEN* \\ Department of Mathematical Sciences, Purdue University School of Science, 1125 East 38th Street, \\ Indianapolis, IN 46205, U.S.A.
}

\begin{abstract}
In this paper the method of signal-flow graphs is used for calculating the Control Coefficients of metabolic pathways in terms of enzyme elasticities. The method is applied to an unbranched pathway $(a)$ without feedback or feedforward regulation and $(b)$ with feedback inhibition of the first enzyme by the last variable metabolite. It is shown that, by using a signal-flow graph, the control structure of a metabolic pathway can be represented in a graphical manner directly from the configuration of the pathway, without the necessity of writing the governing equations in a matrix form. From a signal-flow graph the various Control Coefficients can be evaluated in an easy and straightforward fashion without recourse to matrix inversion or other algebraic techniques. A signal-flow graph also provides a visual framework for analysing the cause-effect relationships of the individual enzymes.
\end{abstract}

\section{INTRODUCTION}

During the past decade Metabolic Control Theory has been used extensively for analysing the regulatory behaviour of metabolic pathways. Metabolic Control Theory was originally proposed by Kacser \& Burns (1973) and Heinrich \& Rapoport (1974), and is based on the notions of Flux Control Coefficients and Concentration Control Coefficients. The Flux (or Concentration) Control Coefficient of an enzyme is defined as the fractional change in metabolic flux (or concentration of a metabolite) in response to a fractional change in enzyme activity. Mathematically, the Flux Control Coefficient of an enzyme $E_{i}$ is given by:

$$
C_{e_{i}}^{J_{j}}=\frac{e_{i}}{J_{j}} \cdot \frac{\partial J_{j}}{\partial e_{i}}
$$

where $J_{j}$ represents any flux in the system and $e_{i}$ is the concentration of the enzyme $\mathrm{E}_{i}$. The Concentration Control Coefficient of the enzyme $E_{i}$ with respect to a metabolite $X_{j}$ is given by the expression:

$$
C_{e_{i}}^{\mathbf{x}_{j}}=\frac{e_{i}}{x_{j}} \cdot \frac{\partial x_{j}}{\partial e_{i}}
$$

$x_{j}$ being the concentration of metabolite $\mathrm{X}_{j}$. The problem of the Control Coefficients is formulated in terms of the enzymic properties known as the Elasticity Coefficients. The Elasticity Coefficient $\left(\epsilon_{\mathbf{x}_{i}}^{v_{i}}\right)$ of an enzyme $\left(\mathrm{E}_{i}\right)$ towards a particular metabolite $\left(X_{j}\right)$ measures the sensitivity of the rate of reaction catalysed by the enzyme with respect to a fractional change in concentration of the metabolite:

$$
\epsilon_{\mathbf{x}_{j}}^{v_{i}}=\frac{x_{j}}{v_{i}} \cdot \frac{\partial v_{i}}{\partial x_{j}}
$$

Here $v_{i}$ is the rate of the reaction catalysed by the enzyme $\mathrm{E}_{i}$. The Control Coefficients are related to the Elasticity Coefficients by means of the so-called Summation and Connectivity Theorems, which represent a system of linear algebraic equations for the unknown Control Coefficients. By solving these equations the
Flux Control Coefficients and Concentration Control Coefficients are evaluated in terms of the Elasticity Coefficients. Several methods have been developed for calculating the Control Coefficients in unbranched, branched and various other pathways. Among these methods the matrix-inversion procedure suggested by Fell \& Sauro (1985) and Westerhoff \& Kell (1987) is most commonly used by researchers. The matrix method consists of expressing the equations governing the Control Coefficients in the form of a matrix of Elasticity Coefficients; the Control Coefficients are derived by inverting this matrix of Elasticity Coefficients. The objective of the present paper is to apply a graphical technique, based on a signal-flow graph or a Mason graph, for the calculation of the Control Coefficients. The method of signal-flow graphs was discovered by Mason (1953) and has been widely used for the analysis of electrical and electronic circuits (Chen, 1971; Chua \& Lin, 1975). In the present paper we apply the signal-flow graphs in a normalized form (see the Appendix).

A major advantage of the method of signal-flow graphs is that the control structure of a metabolic pathway can be represented in a graphical manner directly from the configuration of the pathway, without the necessity of writing the governing equations in a matrix form. From a signal-flow graph the various Control Coefficients can be calculated in an easy and straightforward manner without recourse to matrix inversion or other algebraic techniques. These methods also provide a visual framework for analysing the cause-effect relationships of the individual enzymes in a pathway and thus aid in developing an intuitive understanding of their regulatory functions.

In order to motivate the graphical approach we start with a few definitions. A graph consists of a set of nodes, which are connected by a set of line segments called edges. A signal-flow graph is a weighted directed graph, i.e. each of its edges is given a direction and is assigned a weight (see Fig. 1). The directed edge that originates from a node $i$ and terminates at an adjacent node $j$ is denoted by $(i, j)$. A directed path from a node $i$ to a node $k$ is a series of edges beginning at node $i$ and ending at node $k$, such that each edge is directed from the node just preceding it to the node immediately following it and no edge passes through any

* Present address: Department of Chemical Engineering, California Institute of Technology, Pasadena, CA 91125, U.S.A. 
node more than once. Consider, as an example, the set of edges $(1,2),(2,3)$ and $(3,4)$ in Fig. 1, which constitute a directed path fron node 1 to node 4 . A directed circuit (or simply a loop) is formed by a directed path whose beginning and ending nodes are the same. More precisely, the loop just described is referred to as a first-order loop. Each of Figs. 2(a) to 2(e) depicts a first-order loop. There are also the so-called $n$ th-order loops; an $n$ th-order loop is defined to be a set of $n$ non-touching first-order loops. A set of loops is said to be non-touching if there is neither a node nor an edge in common between any two loops of the set. The gain of a directed path is the product of the weights of all edges constituting that path. Similarly, the gain of a first-order loop is
Coefficients. The symbol $\epsilon_{f}^{i}$ denotes the Elasticity Coefficient of the enzyme $\mathrm{E}_{i}$ with respect to the metabolite $\mathrm{X}_{j}$.

Note that in pathway (A) a metabolite $X_{j}$ acts as a product of the enzyme $E_{j}$ and as a substrate of the enzyme $E_{j+1}$. According to the Connectivity Theorem the Flux Control Coefficients of this pair of contiguous enzymes are in the inverse ratio of their Elasticity Coefficients with respect to the shared metabolite. By using this fact, a signal-flow graph representing the control structure of the pathway (A) can be constructed directly from the configuration of the pathway as follows. We redraw the pathway showing explicitly the Elasticity Coefficients of the enzymes with regard to the respective metabolites:

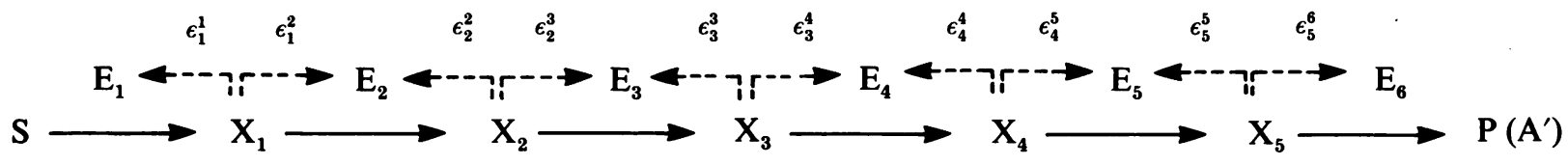

obtained by multiplying the weights of all its edges. The gain of an $n$ th-order loop is equal to the product of the gains of its firstorder loops. Basically, a signal-flow graph is a graphical way of displaying a system of linear algebraic equations. They were originally used by Mason (1953) for analysing the flow of signals through electric circuits. We show below how a signal-flow graph can be utilized to represent and analyse the control structure of a metabolic pathway. We also demonstrate that, by using a signal-flow graph, the Control Coefficients of a given pathway can be readily evaluated in terms of the Elasticity Coefficients.

\section{CONSTRUCTION OF SIGNAL-FLOW GRAPHS}

To illustrate the construction of signal-flow graphs, we first consider the following unbranched pathway with no feedback or feedforward regulation:

$$
\mathrm{S} \stackrel{\mathrm{E}_{1}}{\longrightarrow} \mathrm{X}_{1} \stackrel{\mathrm{E}_{2}}{\longrightarrow} \mathrm{X}_{2} \stackrel{\mathrm{E}_{3}}{\longrightarrow} \mathrm{X}_{3} \stackrel{\mathrm{E}_{4}}{\longrightarrow} \mathrm{X}_{4} \stackrel{\mathrm{E}_{5}}{\longrightarrow} \mathrm{X}_{5} \stackrel{\mathrm{E}_{6}}{\longrightarrow} \mathrm{P}
$$

Here the letters $E$ and $X$, with appropriate subscripts, represent the enzymes and the variable metabolites respectively. The initial precursor and the final product are denoted by $S$ and $P$ respectively; these are assumed to be kept at constant concentration levels. In the present paper we shall confine ourselves to the calculation of Flux Control Coefficients. It should be pointed out, however, that the present methodology can also be applied for the determination of the Concentration Control Coefficients.

According to Metabolic Control Theory, the Flux Control Coefficients are governed by the so-called Summation and Connectivity Theorems. For the above pathway, these theorems are expressed by the following equations (Kacser \& Burns, 1973):

$$
\begin{aligned}
C_{1}^{J}+C_{2}^{J}+C_{3}^{J}+C_{4}^{J}+C_{5}^{J}+C_{6}^{J} & =1 \\
\epsilon_{1}^{1} C_{1}^{J}+\epsilon_{1}^{2} C_{2}^{J} & =0 \\
\epsilon_{2}^{2} C_{2}^{J}+\epsilon_{2}^{3} C_{3}^{J} & =0 \\
\epsilon_{3}^{3} C_{3}^{J}+\epsilon_{3}^{4} C_{4}^{J} & =0 \\
\epsilon_{4}^{4} C_{4}^{J}+\epsilon_{4}^{5} C_{5}^{J} & =0 \\
\epsilon_{5}^{5} C_{5}^{J}+\epsilon_{5}^{6} C_{6}^{J} & =0
\end{aligned}
$$

Eqn. ( $1 a)$ is a statement of the Summation Theorem. The remaining equations in eqn. (1) follow from the Connectivity Theorem applied to each pair of contiguous enzymes. In these equations $C_{i}^{J}(i=1,2,3,4,5,6)$ are the unknown Flux Control
A typical Elasticity Coefficient $\epsilon_{j}^{i}$ is displayed by a broken line with an arrow pointing from the metabolite $X_{j}$ to the enzyme $E_{i}$. The signal-flow graph (see Fig. 1) is drawn beginning with one end of the pathway. For example, let us start with the left end (or the substrate end), i.e. with enzyme $E_{1}$. The Flux Control Coefficient of this enzyme is characterized by node 1 in Fig. 1 ; we refer to this node as the leader node. Next we draw node 2, symbolizing the Flux Control Coefficient of the enzyme $\mathrm{E}_{2}$. The nodes 1 and 2 are joined by a directed edge, as shown in the Figure. To assign a weight to this directed edge, we focus on the segment of the pathway $\left(A^{\prime}\right)$ that consists of the enzymes $E_{1}$ and $\mathrm{E}_{2}$ and the metabolite $\mathrm{X}_{1}$ :

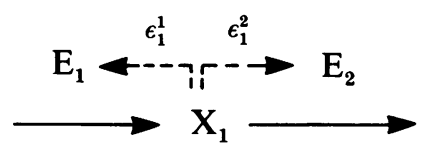

The directed edge $(1,2)$ is given a weight equal to the ratio of the Elasticity Coefficients of the enzymes $E_{1}$ and $E_{2}$ with respect to the adjoining metabolite $X_{1}$, with an appropriate sign. The sign of the weight is decided according to the following rule: if the arrow of the broken line representing the Elasticity Coefficient (e.g., $\left.\epsilon_{1}^{2}\right)$ points along the direction of the forward (i.e. net) reaction of the pathway, then the Elasticity Coefficient is used with its sign unchanged; if, on the other hand, the arrow points in the reverse direction (as with $\epsilon_{1}^{1}$ ), then the Elasticity Coefficient is used with its sign reversed. Furthermore, when the elasticities $\epsilon_{i}^{i}(i=1,2,3,4,5)$ are involved, which represent apparent kinetic orders due to product inhibition, we write $\epsilon_{i}^{i}=-\vec{\epsilon}_{i}^{i}, \vec{\epsilon}_{i}^{i}$ being the absolute value of the Elasticity Coefficient. Thus the directed edge $(1,2)$ is assigned the weight $\bar{\epsilon}_{1}^{1} / \epsilon_{1}^{2}$. The Flux Control Coefficients of the remaining enzymes, namely $E_{3}, E_{4}, E_{5}$ and $E_{6}$, are characterized by the nodes $3,4,5$ and 6 respectively. The weights associated with the directed edges $(2,3),(3,4),(4,5)$ and $(5,6)$ are decided by considering each adjacent pair of enzymes and applying the above rule. Next a directed edge with weight -1 is drawn from each of the nodes (except the leader node) to the leader node, on account of the Summation Theorem [see eqn. ( $1 a$ ) and multiply both sides by -1 ]. Finally we introduce an additional node, node 7 , which is called the source node. A source node is a node from which an edge can only originate, and no edges can enter the node. This source node is assumed to represent a fictitious Flux Control Coefficient $C_{7}^{J}$ of magnitude 1. The fictitious Flux Control Coefficient $C_{7}^{J}=1$ is introduced to comply with the derivation of the Summation Theorem (eqn. 1a), 
if desired, from the signal-flow graph (see the following paragraph). In keeping with the Summation Theorem, a weight of unity is attached to the edge emanating from the source node and terminating at the leader node (see also the Appendix).

The fact that the signal-flow graph of Fig. 1 is indeed consistent with eqn. (1) can be easily established. For example, to derive the $m$ th equation of eqn. (1), we multiply the weight of each incoming edge of node $m$ in the signal-flow graph by the Flux Control Coefficient of the node from which the edge originates; the sum of these products, when added to the negative of the Flux Control Coefficient of node $m$ and equated to zero, yields the $m$ th equation in eqn. (1). Specifically, with node 1 we obtain eqn. (1a); with each remaining node a connectivity relationship consistent with eqns. $(1 b)-(1 f)$ is obtained.

A signal-flow graph for calculating the Concentration Control Coefficients can be constructed in a similar manner. For instance, the Concentration Control Coefficients of the metabolite $X_{1}$ can be derived from Fig. 1 by deleting the edge $(7,1)$ and then adding an edge from the source node 7 to node 2 with a weight of $-1 / \epsilon_{1}^{2}$. Similarly, to evaluate the Concentration Control Coefficients of $X_{3}$ with respect to the various enzymes, we need to introduce

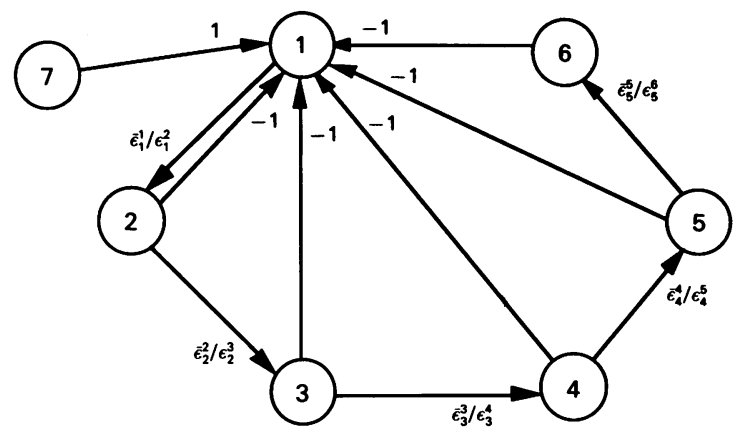

Fig. 1. Normalized signal-flow graph for pathway (A) instead an edge $(7,3)$ with a weight of $-1 / \epsilon_{2}^{3}$, and so on, consistent with the Summation and Connectivity Theorems for the respective Concentration Control Coefficients (Kacser \& Burns, 1973).

Before leaving this section it should be pointed out that there is more than one way of representing the control structure of a metabolic pathway by means of a signal-flow graph. For instance, one may construct a signal-flow graph for the Flux Control Coefficients of pathway (A), beginning with the right end (or the product end) of the pathway, i.e. starting with enzyme $E_{6}$, as shown in Fig. 5. In this Figure node 6 is considered as the leader node; accordingly, an edge is directed to this node from every other node. For comparison, note that each of the edges connecting a pair of nodes $i$ and $i+1(i=1,2,3,4,5)$, except of course the edge $(5,6)$ is in the opposite direction of the corresponding edge in Fig. 1, and carries a weight that is equal to the reciprocal of the weight of the corresponding edge in Fig. 1. Implications of the signal-flow graph of Fig. 5 are discussed in a subsequent section.

\section{EVALUATION OF THE FLUX CONTROL COEFFICIENTS}

By using the signal-flow graph of Fig. 1, a typical Flux Control Coefficient $C_{i}^{J}(i=1,2,3,4,5,6)$ can be deduced from the following formula (see Chua \& Lin, 1975):

$$
C_{i}^{J}=\frac{1}{\Delta} \sum_{k} G_{k i} \Delta_{k i}
$$

with:

$$
\Delta=1-L_{1}+L_{2}-L_{3}+\ldots
$$

Here $L_{1}$ is the sum of the gains of all first-order loops in the signal-flow graph, $L_{2}$ is the sum of the gains of all second-order loops, $L_{3}$ is the sum of the gains of all third-order loops and so on; $G_{k i}$ is the gain of the $k$ th directed path from the source node (a)
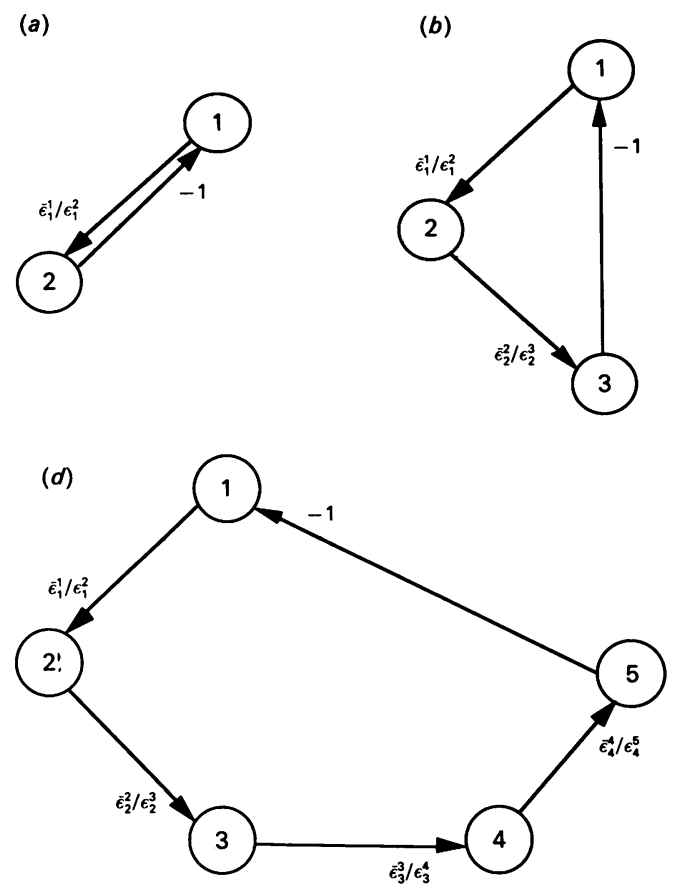
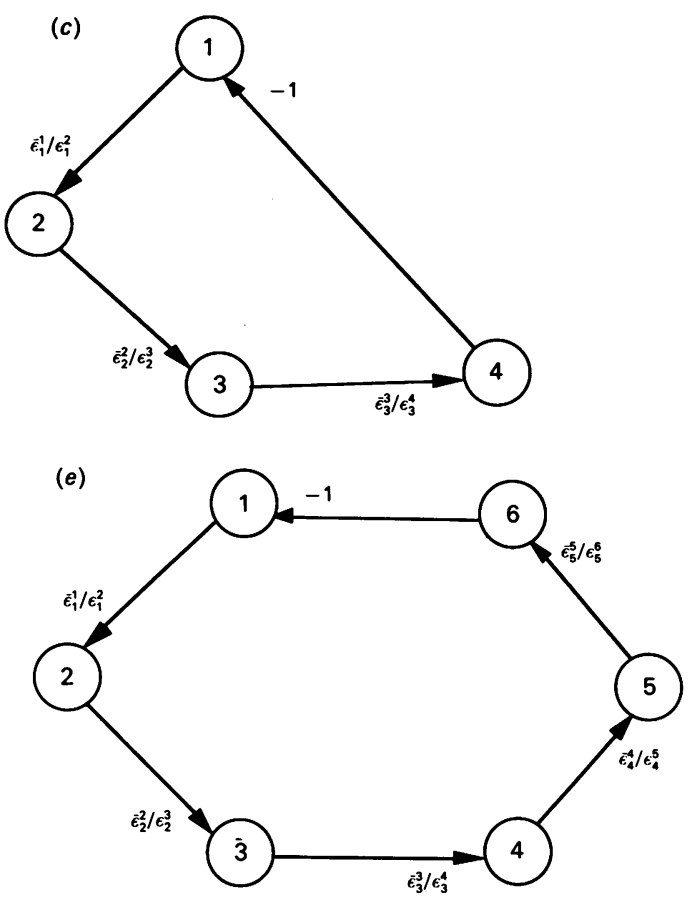

Fig. 2. The five first-order loops in the signal-flow graph of Fig. 1 
to node $i ; \Delta_{k i}$ is the sum of those terms in $\Delta$ (including the first term of 1) which correspond to all the loops in the signal-flow graph that do not touch the $k$ th directed path from the source node to node $i$, and the summation is taken over all directed paths from the source node to node $i$. It is easily seen that the signal-flow graph of Fig. 1 has only first-order loops; these are shown in Fig. 2. By using the gains of these five loops in eqn. (3), we find:

$$
\Delta=1-L_{1}=1+\Delta_{1}+\Delta_{2}+\Delta_{3}+\Delta_{4}+\Delta_{5}
$$

where:

$$
\Delta_{1}=\frac{\bar{\epsilon}_{1}^{1}}{\epsilon_{1}^{2}}, \quad \Delta_{2}=\frac{\bar{\epsilon}_{1}^{1} \bar{\epsilon}_{2}^{2}}{\epsilon_{1}^{2} \epsilon_{2}^{3}}, \quad \Delta_{3}=\frac{\bar{\epsilon}_{1}^{1} \bar{\epsilon}_{2}^{2} \bar{\epsilon}_{3}^{3}}{\epsilon_{1}^{2} \epsilon_{2}^{3} \epsilon_{3}^{4}}, \quad \Delta_{4}=\frac{\bar{\epsilon}_{1}^{1} \bar{\epsilon}_{2}^{2} \bar{\epsilon}_{3}^{3} \bar{\epsilon}_{4}^{4}}{\epsilon_{1}^{2} \epsilon_{2}^{3} \epsilon_{3}^{4} \epsilon_{4}^{5}}, \quad \Delta_{5}=\frac{\bar{\epsilon}_{1}^{1} \bar{\epsilon}_{2}^{2} \bar{\epsilon}_{3}^{3} \bar{\epsilon}_{4}^{4} \bar{\epsilon}_{5}^{5}}{\epsilon_{1}^{2} \epsilon_{2}^{3} \epsilon_{3}^{4} \epsilon_{4}^{5} \epsilon_{5}^{6}}
$$

Thus:

$$
\Delta=1+\frac{\bar{\epsilon}_{1}^{1}}{\epsilon_{1}^{2}}+\frac{\bar{\epsilon}_{1}^{1} \bar{\epsilon}_{2}^{2}}{\epsilon_{1}^{2} \epsilon_{2}^{3}}+\frac{\bar{\epsilon}_{1}^{1} \bar{\epsilon}_{2}^{2} \bar{\epsilon}_{3}^{3}}{\epsilon_{1}^{2} \epsilon_{2}^{3} \epsilon_{3}^{4}}+\frac{\bar{\epsilon}_{1}^{1} \bar{\epsilon}_{2}^{2} \bar{\epsilon}_{3}^{3} \bar{\epsilon}_{4}^{4}}{\epsilon_{1}^{2} \epsilon_{2}^{3} \epsilon_{3}^{4} \epsilon_{4}^{5}}+\frac{\bar{\epsilon}_{1}^{1} \bar{\epsilon}_{2}^{2} \bar{\epsilon}_{3}^{3} \bar{\epsilon}_{4}^{4} \bar{\epsilon}_{5}^{5}}{\epsilon_{1}^{2} \epsilon_{2}^{3} \epsilon_{3}^{4} \epsilon_{4}^{5} \epsilon_{5}^{6}}
$$

Consider first the calculation of $C_{1}^{J}$. Note that there is only one directed path from the source node 7 to node 1 , of weight 1 ; therefore $G_{k i}=1$. Since all the loops of Fig. 2 touch this directed path (via node 1), i.e. there are no non-touching loops, it follows that $\Delta_{k i}=1$. Accordingly, the numerator in eqn. (2) has the value 1 , with the result that $C_{1}^{J}=1 / \Delta, \Delta$ being given by eqn. (6). Next we evaluate $C_{2}^{J}$. The only directed path from the source node to node 2 consists of the edges $(7,1)$ and $(1,2)$. This directed path has a gain of $\bar{\epsilon}_{1}^{1} / \epsilon_{1}^{2}$, which is equal to $\Delta_{1}$. Since there are no nontouching loops with respect to this directed path (see Fig. 2), we obtain $C_{2}^{J}=\Delta_{1} / \Delta$. The remaining Flux Control Coefficients can be calculated in a similar manner. For example, considering the directed path from the source node 7 to node $6, C_{6}^{J}$ is found to be:

$$
C_{6}^{J}=\frac{\Delta_{5}}{\Delta}=\frac{1}{\Delta}\left(\frac{\bar{\epsilon}_{1}^{1} \bar{\epsilon}_{2}^{2} \bar{\epsilon}_{3}^{3} \bar{\epsilon}_{4}^{4} \bar{\epsilon}_{5}^{5}}{\epsilon_{1}^{2} \epsilon_{2}^{3} \epsilon_{3}^{4} \epsilon_{4}^{5} \epsilon_{5}^{6}}\right)
$$

With simple algebraic manipulations these results can be shown to be identical with the corresponding results obtained by the matrix-inversion procedure of Fell \& Sauro (1985), and Westerhoff \& Kell (1987).

We now examine the situation in which the last variable metabolite acts as an allosteric effector and inhibits the first enzyme in pathway (A):
In the above diagram the Elasticity Coefficients of $E_{1}$ and $E_{6}$ with respect to $X_{5}$ are displayed in the manner described in the previous section. According to the nomenclature used above, we may designate the effect of feedback inhibition in pathway (B) by drawing an edge from node 1 to node 6 with a weight $\bar{\epsilon}_{5}^{1} / \epsilon_{5}^{6}$ in Fig. 1, where $\epsilon_{5}^{1}=-\vec{\epsilon}_{5}^{1}, \vec{\epsilon}_{5}^{1}$ being the absolute value of $\epsilon_{5}^{1}$. The signal-flow graph for pathway (B) is shown in Fig. 3. It is clear that Fig. 3 has six first-order loops; five of them are given in Fig. 2 ; the sixth loop is depicted in Fig. 4. Once again there are no higher-order loops in Fig. 3. As before, the Flux Control

Coefficients can be calculated from eqn. (2), where the denominator on the right-hand side is given by the expression:

$$
\hat{\Delta}=1+\frac{\bar{\epsilon}_{1}^{1}}{\epsilon_{1}^{2}}+\frac{\bar{\epsilon}_{1}^{1} \bar{\epsilon}_{2}^{2}}{\epsilon_{1}^{2} \epsilon_{2}^{3}}+\frac{\bar{\epsilon}_{1}^{1} \bar{\epsilon}_{2}^{2} \bar{\epsilon}_{3}^{3}}{\epsilon_{1}^{2} \epsilon_{2}^{3} \epsilon_{3}^{4}}+\frac{\bar{\epsilon}_{1}^{1} \bar{\epsilon}_{2}^{2} \bar{\epsilon}_{3}^{3} \bar{\epsilon}_{4}^{4}}{\epsilon_{1}^{2} \epsilon_{2}^{3} \epsilon_{3}^{4} \epsilon_{4}^{5}}+\frac{\bar{\epsilon}_{1}^{1} \bar{\epsilon}_{2}^{2} \bar{\epsilon}_{3}^{3} \bar{\epsilon}_{4}^{4} \bar{\epsilon}_{5}^{5}}{\epsilon_{1}^{2} \epsilon_{2}^{3} \epsilon_{3}^{4} \epsilon_{4}^{5} \epsilon_{5}^{6}}+\frac{\bar{\epsilon}_{5}^{1}}{\epsilon_{5}^{6}}
$$

Here we have used the symbol $\hat{\Delta}$ to distinguish this pathway from the uninhibited pathway (A). The last term on the right-hand side of eqn. (8) indicates the contribution of feedback inhibition (see Fig. 4).

Let us calculate $C_{1}^{J}$. Note that the directed path from the source node 7 to node 1 in Fig. 3 is the same as the corresponding directed path in Fig. 1, and each of the loops in Fig. 2 and Fig. 4 touches this directed path. Therefore $C_{1}^{J}=1 / \hat{\Delta}$. Similar results hold for $C_{2}^{J}, C_{3}^{J}, C_{4}^{J}$ and $C_{5}^{J}$. We have $C_{2}^{J}=\Delta_{1} / \hat{\Delta}, C_{3}^{J}=\Delta_{2} / \hat{\Delta}$, $C_{4}^{J}=\Delta_{3} / \hat{\Delta}$ and $C_{5}^{J}=\Delta_{4} / \hat{\Delta}$. Observe, however, that there are now two directed paths from the source node 7 to node 6 . Accordingly $C_{6}^{J}$ is given by:

$$
C_{6}^{J}=\frac{1}{\hat{\Delta}}\left(\frac{\bar{\epsilon}_{1}^{1} \bar{\epsilon}_{2}^{2} \bar{\epsilon}_{3}^{3} \bar{\epsilon}_{4}^{4} \bar{\epsilon}_{5}^{5}}{\epsilon_{1}^{2} \epsilon_{2}^{3} \epsilon_{3}^{4} \epsilon_{4}^{5} \epsilon_{5}^{6}}+\frac{\bar{\epsilon}_{5}^{1}}{\epsilon_{5}^{6}}\right)
$$

When the strength of feedback inhibition is very high, i.e. $\bar{\epsilon}_{5}^{1} \rightarrow$ $\infty, C_{6}^{J}$ approaches unity (see eqn. 9), and $C_{1}^{J}=C_{2}^{J}=C_{3}^{J}=C_{4}^{J}$ $=C_{5}^{J}=0$; in this case the last enzyme alone controls the flux through the pathway. It should be pointed out here that under conditions prevailing in vivo a high strength of feedback inhibition is usually not encountered, since a strong feedback inhibition tends to destabilize the steady state of the pathway (Savageau,

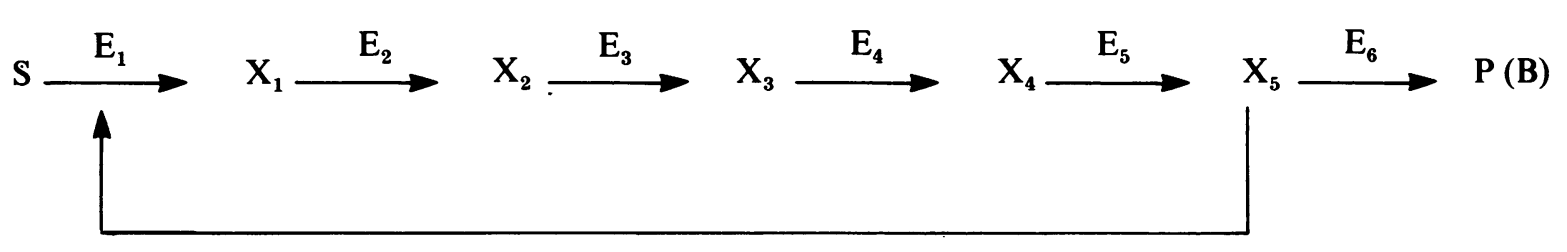

The effect of feedback inhibition in pathway (B) can be represented in a signal-flow graph in the following manner. Let $\epsilon_{5}^{1}$ be the Elasticity Coefficient of enzyme $E_{1}$ with respect to $X_{5}$. For the purpose of drawing the signal-flow graph, we treat the enzymes $E_{1}$ and $E_{6}$ as a pair of contiguous enzymes with $X_{5}$ as the adjoining metabolite, thus:

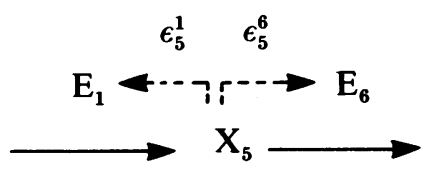

1976). The effect of negative feedback on flux control distribution has been analysed previously by Kacser \& Burns (1973) and Kacser \& Porteous (1987), among others, by using algebraic methods.

\section{FURTHER RESULTS AND DISCUSSION}

We now demonstrate how a normalized signal-flow graph can be used to study the cause-effect relationships of the various enzymes in a metabolic pathway. We examine a few limiting cases such as enzyme saturation, absence of product inhibition and 


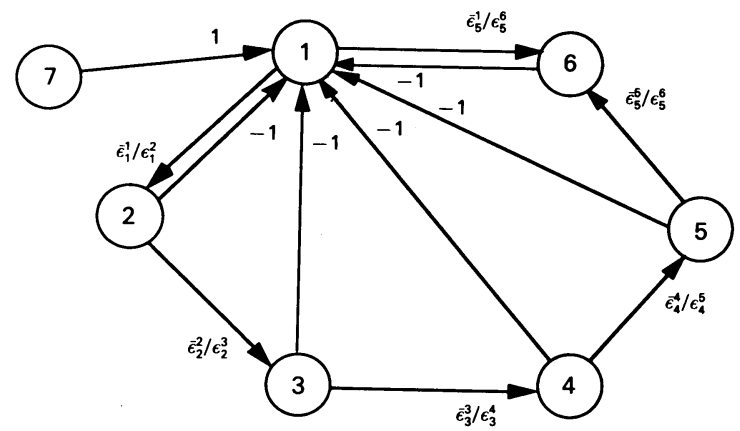

Fig. 3. Normalized signal-flow graph for pathway (B)

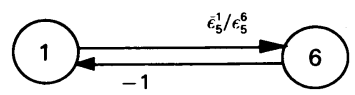

Fig. 4. A first-order loop in Fig. 3, in addition to the first-order loops shown in Fig. 2

chemical equilibrium. Although such extreme conditions do not prevail under physiological conditions, their analysis is helpful for understanding the trend in the regulatory behaviour of the enzymes. The results for these limiting cases can also be derived by inspecting the algebraic form of the governing equations or the solutions from matrix inversion; they can otherwise be obtained in an intuitive manner. To begin with, consider the situation in which the first enzyme in pathway (A) is free of product inhibition, i.e. $\epsilon_{1}^{1}=0$. In this case the directed edge $(1,2)$ in Fig. 1 has zero weight and hence can be deleted. This makes Fig. 1 an acyclic graph, i.e. it has no loops. The denominator $(\Delta)$ on the right-hand side of eqn. (2) is therefore equal to 1 . Furthermore, since there is no directed path from the source node 7 to any of the nodes 2, 3, 4, 5 and 6, the Flux Control Coefficients $C_{2}^{J}, C_{3}^{J}, C_{4}^{J}, C_{5}^{J}$ and $C_{6}^{J}$ must all be zero. There is, however, a directed path of weight 1 from the source node 7 to node 1 ; accordingly $C_{1}^{J}=1$, i.e. the flux through the pathway is regulated solely by the first enzyme. The same conclusion is reached if the second reaction in the pathway (A) is at equilibrium, i.e. $\epsilon_{1}^{2}=\infty$. A situation where $\epsilon_{1}^{1}$ is small and $\epsilon_{1}^{2}$ is large has been reported by Wanders et al. (1984) in the citrulline-synthesizing pathway in isolated rat liver mitochondria. In this pathway the first enzyme, carbamoyl-phosphate synthetase, is very insensitive to inhibition by its product, intramitochondrial carbamoyl phosphate, whereas the second enzyme, ornithine transcarbamoylase, is far from saturation with its substrate. As a consequence, the first enzyme exerts essentially all of the flux control, in keeping with the present results. In a similar fashion it can be shown that, if the third enzyme in pathway (A) is devoid of product inhibition, then $C_{4}^{J}, C_{5}^{J}$ and $C_{6}^{J}$ will be zero and the control will be shared by the first three enzymes in the pathway. In other words, in a linear pathway without feedback or feedforward regulation, no flux control can be exerted by an enzyme that is downstream from an enzyme with no product inhibition (or downstream from a reaction at equilibrium). This is a well-known result, which can be established in an intuitive manner or by other techniques (Fell \& Sauro, 1985).

Consider an analogous situation in pathway (B), i.e. assume that the first enzyme in this pathway has no product inhibition (or the second reaction is at equilibrium). It is easily seen from Fig. 3 that under these circumstances $C_{2}^{J}=C_{3}^{J}=C_{4}^{J}=C_{5}^{J}=0$, and the control of flux is distributed between the first and the last

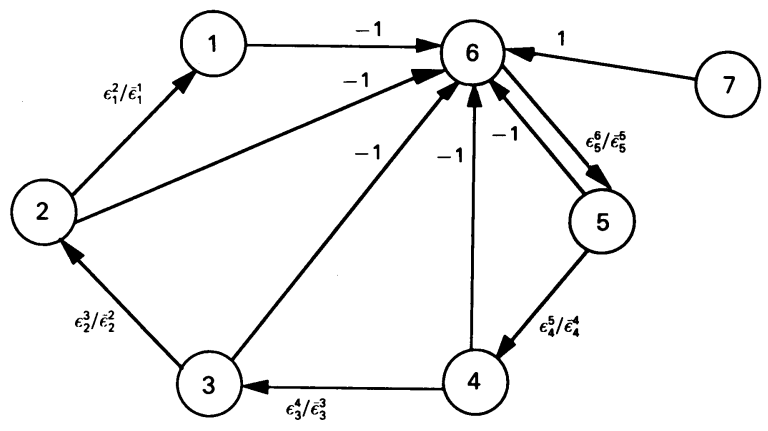

Fig. 5. Normalized signal-flow graph for pathway (A) for studying the effects of enzyme saturation

enzymes. If, instead, the third enzyme in this pathway is insensitive to product inhibition, then Fig. 3 shows that the enzymes $E_{1}, E_{2}, E_{3}$ and $E_{6}$ will share the control while $E_{4}$ and $E_{5}$ remain ineffective.

It should be pointed out that, if any of the enzymes in pathway (A) or pathway (B) is completely saturated, its effect on the control of flux by the various enzymes cannot be ascertained directly from the signal-flow graph of Fig. 1 or Fig. 3, since under these circumstances the respective edge weights become infinite. Of course, one can calculate the expressions for all the Flux Control Coefficients, say for pathway (A), from Fig. 1 by means of eqns. (5) and (6) and substitute a zero for the Elasticity Coefficient of the saturated enzyme in those expressions. Alternatively, these cause-effect relationships can be analysed from the normalized signal-flow graph of Fig. 5. The method of construction of Fig. 5 was described in a previous section. From this Figure the Control Coefficients $C_{i}^{J}(i=1,2,3,4,5,6)$ can be calculated directly by use of eqn. (2). For instance, considering the directed path of weight 1 from the source node 7 to node 6 , and taking into account the five first-order loops in this Figure, $C_{6}^{J}$ is found to be:

$$
C_{6}^{J}=1 /\left(1+\frac{\epsilon_{5}^{6}}{\bar{\epsilon}_{5}^{5}}+\frac{\epsilon_{4}^{5} \epsilon_{5}^{6}}{\bar{\epsilon}_{4}^{4} \bar{\epsilon}_{5}^{5}}+\frac{\epsilon_{3}^{4} \epsilon_{4}^{5} \epsilon_{5}^{6}}{\bar{\epsilon}_{3}^{3} \bar{\epsilon}_{4}^{4} \bar{\epsilon}_{5}^{5}}+\frac{\epsilon_{2}^{3} \epsilon_{3}^{4} \epsilon_{4}^{5} \epsilon_{5}^{6}}{\bar{\epsilon}_{2}^{2} \bar{\epsilon}_{3}^{3} \bar{\epsilon}_{4}^{4} \bar{\epsilon}_{5}^{5}}+\frac{\epsilon_{1}^{2} \epsilon_{2}^{3} \epsilon_{3}^{4} \epsilon_{4}^{5} \epsilon_{5}^{6}}{\bar{\epsilon}_{1}^{1} \bar{\epsilon}_{2}^{2} \bar{\epsilon}_{3}^{3} \bar{\epsilon}_{4}^{4} \bar{\epsilon}_{5}^{5}}\right)
$$

With a slight manipulation, this result can be shown to be identical with the expression for $C_{6}^{J}$ obtained above in eqn. (7). To see the effect of enzyme saturation on flux control, consider the situation when the enzyme $E_{3}$ is saturated, i.e. $\epsilon_{2}^{3}=0$. In this case there will be a directed path from the source node 7 to each of the nodes $3,4,5$, and 6 , but the nodes 1 and 2 cannot be reached from the source node. Accordingly $C_{1}^{J}$ and $C_{2}^{J}$ will both be zero and the control of flux will be distributed among the enzymes $E_{3}, E_{4}, E_{5}$ and $E_{6}$. In general, it can be shown that no control is exerted by enzymes that are upstream from a saturated enzyme in this pathway. This result is also intuitively clear. Effects of enzyme saturation can be analysed in a similar fashion in pathway (B), which possesses feedback inhibition.

From a practical standpoint, it may sometimes be sufficient to know the relative magnitudes of the Flux Control Coefficients of the various enzymes in a given pathway, rather than their actual values. These can be assessed directly from the corresponding signal-flow graph as follows. Consider the pathway (B), for which the normalized signal-flow graph is given in Fig. 3. Suppose that we want to find the ratio of the Flux Control Coefficient of any of the enzymes $E_{2}, E_{3}, E_{4}, E_{5}$ and $E_{6}$ to that of the first enzyme. This can be accomplished by converting node 1 


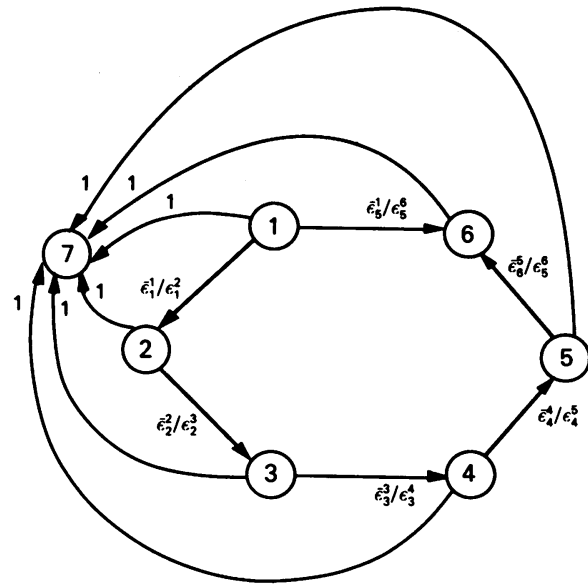

Fig. 6. Conversion of node 1 into a source node in the signal-flow graph of Fig. 3

in Fig. 3 into a source node and transforming the original source node 7 into an ordinary (non-source) node (or by inverting the directed edge from the source node to node 1). I now describe the node-conversion (or edge-inversion) procedure in general. Let $i$ be a source node and $j$ be an adjacent ordinary node in a normalized signal-flow graph. Consider that the directed edge $(i, j)$ has a weight $w_{i j}$ associated with it. To convert the node $j$ into a source node, we first move all the edges having terminal node $j$ to node $i$. Next, we move the initial node of the self-loop $(i, i)$ thus created at node $i$ to node $j$; in other words, the directed edge $(i, j)$ in the original signal-flow graph becomes the 'inverted' edge $(j, i)$ in the converted graph; this edge is assigned a weight equal to $1 / w_{i j}$. Finally, we multiply the weights of all the other edges, whose terminal nodes have been moved to node $i$, by $-1 / w_{i j}$. From the converted signal-flow graph, the ratio of the Flux Control Coefficient of an enzyme, say $\mathrm{E}_{p}$, to that of $\mathrm{E}_{j}$ can be deduced from the following formula:

$$
\frac{C_{p}^{J}}{C_{j}^{J}}=\frac{1}{\tilde{\Delta}} \sum_{k} \tilde{G}_{k j p} \tilde{\Delta}_{k j p}
$$

where

$$
\tilde{\Delta}=1-M_{1}+M_{2}-M_{3} \ldots
$$

$M_{1}$ being the sum of the gains of all first-order loops, $M_{2}$ the sum of the gains of all second-order loops and so on in the converted signal-flow graph; $\tilde{G}_{k j p}$ is the gain of the $k$ th directed path from node $j$ to node $p$ in this graph, and $\tilde{\Delta}_{k j p}$ is the sum of those terms in $\tilde{\Delta}$ (including the first term of 1 ) which correspond to loops that do not touch the $k$ th directed path just described. If, in the original signal-flow graph, the source node $i$ is connected to node $j$ via a directed path consisting of more than one edge, then to convert the node $j$ into a source node we must invert all the edges in that directed path successively.

By following the above procedure, node 1 of Fig. 3 is converted into a source node; the resulting signal-flow graph is shown in Fig. 6. Since there are no loops in this Figure, $\tilde{\Delta}$ must be equal to 1 . To calculate $C_{2}^{J} / C_{1}^{J}$, for example, observe that there is only one directed path from node 1 to node 2 , with a weight $\bar{\epsilon}_{1}^{1} / \epsilon_{1}^{2}$, and there are no non-touching loops with respect to this directed path. Therefore $C_{2}^{J} / C_{1}^{J}=\bar{\epsilon}_{1}^{1} / \epsilon_{1}^{2}$. The ratios $C_{3}^{J} / C_{1}^{J}, C_{4}^{J} / C_{1}^{J}$ and $C_{5}^{J} / C_{1}^{J}$ can be similarly found. These ratios can also be obtained from the connectivity relationships for each pair of contiguous enzymes. To calculate $C_{6}^{J} / C_{1}^{J}$, however, notice that there are two directed paths from node 1 to node 6; furthermore, since Fig. 6 is acyclic, clearly there are no non-touching loops to either of these directed paths; as a result, we find:

$$
\frac{C_{6}^{J}}{C_{1}^{J}}=\frac{\bar{\epsilon}_{1}^{1} \bar{\epsilon}_{2}^{2} \bar{\epsilon}_{3}^{8} \bar{\epsilon}_{4}^{4} \bar{\epsilon}_{5}^{5}}{\epsilon_{1}^{2} \epsilon_{2}^{3} \epsilon_{3}^{4} \epsilon_{4}^{5} \epsilon_{5}^{6}}+\frac{\bar{\epsilon}_{5}^{1}}{\epsilon_{5}^{6}}
$$

If it is desired to find the magnitudes of the various Flux Control Coefficients, say relative to $C_{3}^{J}$, then we need to convert node 3 in Fig. 3 into a source node. To do this, the directed path from the source node to node 3 should be inverted by inverting the directed edges $(7,1),(1,2)$ and $(2,3)$ successively in the manner described above.

\section{CONCLUDING REMARKS}

We have used normalized signal-flow graphs for calculating the Flux Control Coefficients of unbranched metabolic pathways. These graphs can be constructed directly from the configuration of the pathway, without having to write the summation and connectivity relationships in a matrix form. They bring out the fact that the ratios of elasticities of each pair of contiguous enzymes are important in determining the Control Coefficients. They can also be utilized for studying the cause-effect relationships of the various enzymes in a given pathway. It is apparent that the present methodology can be applied to more complex pathways such as those containing branches and cycles.

Signal-flow graphs can also be drawn from a matrix formulation of the Control Coefficients of a metabolic pathway. For completeness, the method of constructing a signal-flow graph from the associated matrix is described in the Appendix. Finally it should be pointed out that alternative approaches for control analysis of metabolic pathways have been given by Reder (1988) and Hofmeyr (1989).

I thank the anonymous referees for helpful suggestions that significantly improved the presentation in this paper.

\section{REFERENCES}

Chen, W. K. (1971) North-Holland Series in Applied Mathematics and Mechanics, vol. 13: Applied Graph Theory, North-Holland, Amsterdam

Chua, L. O. \& Lin, P. M. (1975) Computer-Aided Analysis of Electronic Circuits: Algorithms and Computational Techniques, Prentice-Hall, Englewood Cliffs

Fell, D. A. \& Sauro, H. M. (1985) Eur. J. Biochem. 148, 555561

Heinrich, R. \& Rapoport, T. (1974) Eur. J. Biochem. 42, 89102

Hofmeyr, J. H. S. (1989) Eur. J. Biochem. 186, 343-354

Kacser, H. \& Burns, J. A. (1973) Symp. Soc. Exp. Biol. 27, 65104

Kacser, H. \& Porteous, J. W. (1987) Trends Biochem. Sci. 12, 2 14

Mason, S. J. (1953) Proc. IRE 41, 1144-1156

Reder, C. (1988) J. Theor. Biol. 135, 175-202

Savageau, M. A. (1976) Biochemical Systems Analysis: A Study of Function and Design in Molecular Biology, Addison-Wesley, Reading

Wanders, R. J., Van Roermund, C. W. T. \& Meijer, A. J. (1984) Eur. J. Biochem. 142, 247-254

Westerhoff, H. V. \& Kell, D. B. (1987) Biotechnol. Bioeng. 30, 101107 


\section{APPENDIX}

We describe below how a signal-flow graph can be constructed from a matrix representation of the Summation and Connectivity Theorems given by eqn. (1) of the main paper. For this purpose we write eqn. (1) of the main paper in the matrix form $\mathbf{E} \cdot \mathbf{C}=\mathbf{0}$, as shown below:

$$
\left|\begin{array}{rrrrrrr}
-1 & -1 & -1 & -1 & -1 & -1 & 1 \\
\bar{\epsilon}_{1}^{1} / \epsilon_{1}^{2} & -1 & 0 & 0 & 0 & 0 & 0 \\
0 & \bar{\epsilon}_{2}^{2} / \epsilon_{2}^{3} & -1 & 0 & 0 & 0 & 0 \\
0 & 0 & \bar{\epsilon}_{3}^{3} / \epsilon_{3}^{4} & -1 & 0 & 0 & 0 \\
0 & 0 & 0 & \bar{\epsilon}_{4}^{4} / \epsilon_{4}^{5} & -1 & 0 & 0 \\
0 & 0 & 0 & 0 & \bar{\epsilon}_{5}^{5} / \epsilon_{5}^{6} & -1 & 0
\end{array}\right|\left|\begin{array}{c}
C_{1}^{J} \\
C_{2}^{J} \\
C_{3}^{J} \\
C_{4}^{J} \\
C_{5}^{J} \\
C_{6}^{J} \\
C_{7}^{J}
\end{array}\right|=\left|\begin{array}{l}
0 \\
0 \\
0 \\
0 \\
0 \\
0
\end{array}\right|
$$

The above equation is obtained from eqn. (1) of the main paper in the following manner. In the $k$ th equation $(k=1,2, \ldots, 6)$ every term is divided by the negative of the coefficient of $C_{k}^{J}$ appearing in that equation. The vector $\mathbf{C}$ contains the six Flux
Control Coefficients $C_{i}^{J}(i=1,2,3,4,5,6)$ and a fictitious variable $C_{7}^{J}$, which is set equal to 1 . The coefficient matrix $\mathbf{E}$ consists of the ratios of the Elasticity Coefficients of each pair of contiguous enzymes, and is augmented by a rightmost column

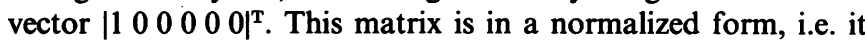
has the elements $E_{i i}$ (for $i=1,2, \ldots, 6$ ) equal to -1 . The terminology 'normalized signal-flow graph' is derived from this matrix structure. From the matrix $\mathbf{E}$, the signal-flow graph of Fig. 1 of the main paper may be drawn by using the following convention. Each node $i$ except node 7 represents a Flux Control Coefficient, $C_{i}^{J}$; the source node 7 corresponds to $C_{7}^{J}=1$. An element $E_{i j}$, with $i \neq j$, gives rise to a directed edge from node $j$ to node $i$, carrying a weight $E_{i j}$. The elements $E_{i i}$ (which are all equal to -1 ) do not contribute to any edges in the normalized signal-flow graph. Clearly the method of construction of a signalflow graph used in the text is consistent with that derived from this matrix formulation. A characteristic feature of a normalized signal-flow graph such as the one shown in Fig. 1 of the main paper is that contains no self-loops, i.e. no edge by itself forms a loop around any node. 